\title{
Internet Auctions with Many Traders
}

\author{
Michael Peters and Sergei Severinov \\ University of Toronto and University of Wisconsin
}

First Version Feb/01 This version June 8, 2001

\begin{abstract}
A multi-unit auction environment similar to Ebay is studied. Sellers who wish to sell a single unit of a homogenous good set reserve prices for their own independently run auctions. Buyers who hope to acquire a single unit bid as often as they like in a dynamic second price auction. When the number of buyers and sellers is large but finite, there is a Bayesian equilibrium for this completely decentalized trading procedure in which the ex post efficient set of trades occurs at a uniform trading price. Remarkably, the strategy rules that buyers and sellers use in this equilibrium are very simple. They do not depend in any way on beliefs, or on the number of buyers and sellers.
\end{abstract}

One of the central features of auction theory is the centralized nature of trade. Specifically, the seller, or auctioneer, establishes trading rules centrally processes information contained in messages sent by buyers and adjusts prices. Some recent examples include algorithms developed by Gul and Stacchetti (2000) and Ausubel (2000), both of which support efficient competitive trade as a Bayesian Nash equilibrium. Theory is less well developed in the case of multilateral exchanges, like the stock exchange and some business to business sites on the internet, yet centralized processing of demand and supply information is a common feature. ${ }^{1}$ It is natural to think in these terms since standard competitive reasoning is typically explained using a fictitious auctioneer who collects demand and supply information then uses some sort of tatonement process to compute market clearing prices. $^{2}$

The theoretical literature that does exist on multilateral trade follows this lead by assuming that messages like bid and ask prices are centrally processed and used to compute efficient trades. Double auctions, for example Wilson

\footnotetext{
${ }^{1}$ For example, the NTE truck exchange (http://www.nte.net) allows truckers with excess carrying capacity to match with shippers who have small and unusual packages to send. The exchange does not use auctions, but instead uses what they call 'market based pricing' to complete the trade. Apparently the 'commodities' used in this exchange are so narrowly defined that mechanisms similar to a double auction that use only demand and supply for a single 'commodity' to determine the price attract too few buyers and sellers, so supply and demand information for related routes and times is aggregated and used to generate market prices.

${ }^{2}$ For example, Herings (1997).
} 
(1985), are known to be incentive efficient. Rustichini, Satterthwaite, and Williams (1994) (following previous work by Satterthwaite and Williams (1089)) show that surplus lost by trading in centralized double auctions shrinks to zero very quickly as the number of traders gets large. Older experimental work by, for example, (Smith (1962)) seems to confirm these theoretical predictions.

At first glance, the trading technology offered by the internet appears to enhance this trend toward centralization since computers make it easier to assemble and process large amounts of information. Large trading institutions such as Ebay,Ubid, and other business-to-business exchanges have emerged, ${ }^{3}$ and are growing quickly. For example, Ebay had 18.9 million registered users as of April 2001. Total sales volume at Ebay in 2000 was $\$ 5$ bln. and this number is expected at least to double in 2001 (see Morneau (2001)). The volume of trade at business-to-business marketplaces has grown from $\$ 5$ bln. in 1999, to $\$ 43 \mathrm{bln}$. in 2000 and, according to ActivMedia Research, is expected to reach $\$ 263$ bln. in 2001 (see Wilson and Mullen (2000), Morneau (2001)).

On closer inspection, the notion that electronic trade promotes centralization of trade is less convincing. Though Ebay certainly presents a focal location for trade, Ebay itself does not do the processing of demand and supply information that occurs in a double auction, or in the single auctioneer mechanisms of Gul and Stacchetti (2000), orAusubel (2000). It is up to sellers to choose their own reserve prices and buyers to decide where to submit bids. Experienced traders apparently buy and sell in many locations. For example, it is possible to buy computers on Ebay, but they can also be purchased on line at suppliers own websites and at a variety of on line retail locations. Suppliers who do try to sell on Ebay, typically also offer their computers on other on line trading sites like Amazon, or Ubid.

The same technology that makes it possible to process large volumes of information also promotes decentralized trade because it makes it possible for individual traders to collect dispersed information about prices and availability and react to it. For example, one could easily imagine that the robot guided bidding on Ebay could be carried out on a host or small private servers (owned by sellers) which simply enforced the trading rules used at the larger institution. Arguably the widespread use of relatively low power personal computers would seem to enhance decentralized decision making as much as it does central computation. This may be one reason that institutions like Ebay are so successful - they leave the responsibility for coordinating trade up to traders themselves.

Whether decentralized trade works or not depends on whether the algorithm used to compute prices and efficient trades can be broken up into bits that can be solved by individual traders, and whether traders have any incentive to follow the rules of the algorithm once this occurs. This issue has been addressed many times before, for example, it has been shown that in the context of random matching - bargaining may or may not produce competitive outcomes when trade frictions are small (see, for example, Rubinstein and Wolinsky (1990)). In

\footnotetext{
${ }^{3}$ The examples of large-scale business-to-business internet exchanges include CheMatch and Chem Connect (chemical industry), Covisint (auto parts), MetalSpectrum (aluminum, stainless steel, copper, iron, and other metals).
} 
this paper, we extend this line of inquiry into an environment that has many of the characteristics of internet auctions and exchanges. Frictions are small and the appropriate way to match buyers and sellers is not obvious.

First, in regards to breaking up the algorithm into smaller parts, we allow sellers to (independently) run ascending price auctions similar to those that occur on Ebay. Buyers submit bids at these auctions, but when these bids are unsuccessful, we allow buyers to adjust their bids and move between sellers costlessly. We then provide a 'simple' bidding rule that buyers can use to select among sellers' auctions and choose their bids. This bidding rule uses information on publicly observable prices, and on the last bid submitted at each auction. Otherwise it is independent of buyers' beliefs about the valuations of the other buyers and on the number of other buyers bidding. When buyers follow this bidding rule, all trades occur at a uniform price that coincides with the price in a 'sellers bid double auction' where all bids and asks are processed in the centralized way ${ }^{4}$ and sellers' ask prices are equal to their reserve prices. If the sellers' reserve prices happened to be equal to their true valuations, then the uniform trading price would be equal to the lowest market clearing price.

Then we show that the bidding rule that we specify constitutes a Bayesian Nash (continuation) equilibrium for buyers given the reserve prices of sellers. So not only does the algorithm parcel out the problem of computing equilibrium prices and trades, but buyers involved have the correct incentive to follow this algorithm.

In the second part of the paper, we show that there is a large but still finite number of buyers and sellers such that it is a Bayesian equilibrium for sellers to set their reserve prices equal to their true costs. So in this sense our decentralized market along with the simple bidding rule we describe for sellers always supports efficient trade at a uniform price when the market is large. ${ }^{5}$ So the 'competitive outcome' is completely decentralized.

The significance of the bidding procedure we descibe is not so much that it generates a competitive outcome, for it is known that there are many algorithms that will accomplish this. The algorithms described by Gul and Stacchetti (2000) and Ausubel (2000) are examples in the single seller case. Roth and Sotomayor (1990) describe an auction like mechanism that generates competitive trade in the environment we study. Demange (1982) and Leonard (1983) study the incentive properties of this mechanism. These procedure are not decentralized in the sense that ours is, since they require that a centralized auctioneer collect information and change prices ${ }^{6}$ The interest in our procedure

\footnotetext{
${ }^{4}$ The sellers' bid double auction is described in Satterthwaite and Williams (1089).

${ }^{5}$ We get stronger results in this regard than Rustichini, Satterthwaite, and Williams (1994) concerning the existence and efficiency of equilibria because we assume that there is a finite set of feasible valuations rather than a continuum. This assumption also allows us to get around the impossibility result of Myerson and Satterthwaite (1983).

${ }^{6}$ Roughly each buyer tells the auctioneer which sellers he would be willing to purchase from at the current set of prices. The auctioneer checks to see whether a matching of buyers to sellers is feasible given this information. If not prices are increased a bit at all the sellers where there is an excess demand. Buyers could send messages to each of the sellers in their 'demand sets' informing them they are willing to trade, but sellers who have an excess demand can't
} 
stems from the fact that the price adjustments are done by traders themselves without any need for centralized intervention. The strategy rules that traders use to conduct themselves are simple. Buyers need to know only the current set of prices as well as whether or not the last bid with each seller (if there was one) was successful (in the sense that the buyer who submitted the bid became high bidder).

Apart from its theoretical value, this result has potentially important practical implications since a decentralized market is less prone to technological failures $^{7}$. We emphasize that our decentralization result does not require that traders themselves carry out the sophisticated calculations that otherwise might have to be done by the mechanism designer intermediating the trades, or by buyers and sellers attempting to foresee future trading prices in a rational expectations model. The strategies that buyers and sellers use 'work' independently of the tastes, beliefs about costs or valuations and, for buyers, even the number of traders who participate in the process. Thus, traders need very little information in order to carry out their equilibrium strategies.

Despite the fact that the market is completely decentralized in our model, the rules that govern exchange are very similar to the ones that are used on wellknown internet auction markets since they are themselves quite decentralized. In this sense, our model gives some insight into the workings of markets like Ebay $^{8}$ and other business-to-business marketplaces.

If bidders follow the strategies we descibe, predictable patterns of bids and pricing will emerge. For example, the winning bid in our model is not necessarily the last bid. However, because the bidders push up the standing bids slowly, the winning bid will normally be submitted towards the end of an auction. It would be rare in our mechanism for the winning bid to be submitted early in the auction (in the sense that there are many bids submitted after it). There is strong evidence that Ebay auctions are won by late bidders (Zheng (2001) or Roth and Ockenfels (2000)). High valuation bidders who follow Ebay's advice to bid their true valuations early will win the auctions they participate in whether they bid early or late.

It is readily apparent in our model why buyers do not want to follow Ebay's advice and bid their true valuations right away, leaving the robots to compute the prices later. Buyers who do so may be accidentally trapped into paying a higher price than they need to when a high valuation buyer bids against them. Incrementing the standing bid as slowly as possible (by submitting the minimum acceptable bid) avoids this.

tell whether a feasible match is possible or whether they should raise price without a signal from the auctioneer.

${ }^{7}$ The Toronto Stock Exchange for example, has suffered continuing technical problems that have shut down its computerized trading system repeatedly and resulted in millions of dollars in trading losses.

8 ebay is a more centralized mechanism than the one we have in mind since the bidding is actually processed on ebay servers. It is not beyond the realm of possibility to imagine that sellers conduct the same auctions on their own servers. The boundaries of the market defined by the internet go far beyond the confines of a single server. Many companies who sell on ebay (sun computers is an example) use ebay as one of many different on line trading centers. 
Another characteristic of the equilibrium is that winning bidders will tend to do more bidding than losing bidders (not necessarily all in the same auction). There is some preliminary evidence that activity and success are correlated consistent with our prediction. Our model predicts strong negative correlation between seller's reservation price and bidding activity. Seller's with high reservation prices should get few bids. However, at least to the extent that the commodities being sold are substitutes, our model also predicts that the units with high and lower reservation price should end up trading at the same price so the correlation between trading price and the seller's reserve price should be very weak.

There are (at least) two branches in the literature that are closely related to our paper. Our paper follows the lead of the literature on competing auctions McAfee (1993), Peters (1997), Peters and Severinov (1997), Hernando-Veciana (2000). The flavor of the results in that literature is similar to ours - when there are many competing auctions, equilibrium indirect mechanisms are independent of the characteristics of demand - second price auctions with reserve prices set equal to costs are best mechanisms for sellers independent of their beliefs. We provide the same kind of result here since sellers use second price auctions and set their reserve prices equal to their costs (though sellers are only free to choose a reserve price in our model, they cannot use mechanisms other than auctions). The difference is that in the previous literature the cost of bidding is implicitly very high in the sense that buyers are able to submit only a single bid. As a consequence, the equilibrium outcomes in these models are not ex post efficient. Coordination problems are large enough that there are unrealized gains to trade. In our model bidders are allowed to submit as many bids as they like. In effect, this costless communication allows buyers to get around the coordination problems that arise in these earlier models.

We have mentioned already the connection between our work and the pricing algorithms of Gul and Stacchetti (2000), Ausubel (2000) and Roth and Sotomayor (1990). Our procedure is closer in spirit to the 'deferred acceptance' algorithms of Gale and Shapley (1962) and Kelso and Crawford (1982) (the latter studies the case with transferable utility). These algorithms have the same decentralized feature that ours does in that proposal acceptance decisions can be left up to individual traders. It is known that the deferred acceptance algorithms are not information proof when utility is non-transferable. Less is known about the incentive properties of these algorithms with transferable utility. We do not address the incentive properties of this kind of algorithm in general, rather we focus on Bayesian Nash equilibria when the number of traders is large and valuations are drawn independently to show that efficient trade is supported.

Our arguments can be broken up into two parts. In the next section we describe our model. This is followed with a description of the equilibrium strategies for buyers. Once we show that on the equilibrium path outcomes are equivalent to those that occur in a seller's bid double auction, the analysis of sellers' equilibrium strategies is then standard, but analytically demanding. In fact, our argument shows that sellers' bid double auctions have efficient equilibria 
when there is a finite set of valuations. This could be viewed as an independent contribution of the paper.

\section{The Model}

There are $n$ sellers and $m$ buyers trading in a market. The number of buyers and sellers is arbitrary in our model, and we do not assume that there are more buyers than sellers. Each seller has one unit of a homogeneous good, while each buyer has an inelastic demand for one unit of this good. Buyers' valuations and sellers' costs are private information and are distributed on the grid $\mathcal{D} \equiv\{\underline{d}, \underline{d}+d, \underline{d}+2 d, \ldots \bar{d}\}$ that has a step size $d>0$. Let $F(\cdot)$ and $G(\cdot)$ be the probability distributions from which buyers' and sellers' valuations respectively are drawn. Our results on equilibrium bidding behavior by buyers are independent of whether or not buyers' valuations are correlated, though our equilibrium for the sellers' part of the game relies on independence. A buyer with valuation $v$ who wins a single auction at a price $p$ gets surplus $v-p$. A buyer who wins more than one auction gets no additional utility from the additional units of output (so his payoff will fall because he has to pay for the additional units). A sellers with cost $c$ who sells at price $p$ get surplus $p-c$.

Trade is organized in the following way. At first, sellers simultaneously announce reserve prices for their auctions. Thereafter buyers arrive sequentially. When a new buyer arrives, he submits a bid at whichever of the sellers' auctions he likes best. Buyers are required to submit bids in the grid $\mathcal{D} .{ }^{9}$ The grid size $d$ could be thought of as the minimum bid increment. When the seller receives a bid, she publishes a number called her standing bid which is equal to the second highest bid that she has received, or her reserve price if she has not received more than 1 bid. Each seller immediately updates her standing bid announcement when her standing bid changes. As in most existing on-line auctions, we also assume that the identity of the winning bidder is known at all times ${ }^{10}$.

We assume throughout that the second-highest bid means second highest bid submitted by a distinct bidder. The standing bid is assumed to remain unchanged if the high bidder revises his bid. A new bid submitted at a seller's auction must always exceed that seller's current standing bid. If two or more bidders have submitted the same high bid, then the buyer who was the first to submit this bid is declared the high bidder. The standing bid in this case is equal to the high bidder's bid.

After a new bid is submitted, each buyer in order of his or her entry into the market is given the opportunity either to submit a new bid (not necessarily with the same seller) or pass. Once each buyer in the market chooses to pass, a new buyer enters. After all buyers have entered the market, the bidding process

\footnotetext{
${ }^{9}$ This assumption is natural in view of our interpretation that the grid on which the traders' valuations are distributed is determined by the minimal monetary unit.

${ }^{10}$ As will be show later, this assumption, or more precisely, the observability of the change in the identity of the winning bidder is important for the uniform price result. When such changes are not observable, price dispersion may occur
} 
continues as bidders update their bids one after another. The order of bidding at this stage is the same as the order of entry with the last bidder followed by the first bidder and so on. The bidding continues until all buyers pass. Then the high bidder at each seller trades at the final standing bid with that seller.

These rules approximate the trading rules on Ebay and on the Amazon auction site (which differs from Ebay primarily in that auctions do not have a definite ending date (see Roth and Ockenfels (2000))). The key property of the auction rules is that they generate a type of the second price auction in which the high bid is never observed.

Despite the second price nature of the auction mechanism, the presence of multiple auctions implies that it is not a dominant strategy for buyers to bid their true valuations when they start bidding (despite the advice offered on the ebay website). To see this, consider the data in Figure 1. For the moment, ignore the point $b_{3}$, and suppose that there are two buyers with true valuations $b_{1}$ and $b_{2}$ and two sellers who announce reserve prices $s_{1}$ and $s_{2}$. Assume that the buyer with valuation $b_{1}$ enters first and expects the other buyer to bid his true valuation wherever he decides to bid.

To show that it is not a dominant strategy, we only need to show that valuation bidding can be strictly improved upon for some strategy of buyer 2, so suppose that buyer 2 is expected to bid his valuation where the standing bid is lowest. Buyer 1 could bid her valuation with seller 2. She should then expect buyer 2 to bid his valuation with seller 1 who has a lower reserve price. No matter what bid buyer 2 submits, buyer 1 will trade at price $s_{2}$. Buyer 1 can strictly increase her expected payoff by initially bidding $s_{2}$ at seller 1 , provided she believes that buyer 2 has a valuation below $s_{2}$ with strictly positive probability.

So if buyer 1 is going to start off bidding her valuation, she will bid with seller 1 . When buyer 2 enters, the standing bid with seller 1 remains at $s_{1}$ because seller 1 has yet to receive a second bid. So by the rule that buyer 2 is supposed to be using, buyer 1 should expect buyer 2 to bid against her provided he bids at all.

If buyer 2 bids at or below $s_{2}$, then buyer 1's initial strategy works out fine, and she trades at a price at or below $s_{2}$. If buyer 2 submits any bid above $b_{2}$, then buyer 1 will be displaced as high bidder, and will trade at price $s_{2}$ with seller 2 - again a reasonable outcome. The difficulty arises when buyer 2 submits a bid strictly above $s_{2}$ but strictly below $b_{2}$. In this case, buyer 1 will pay a price strictly above $s_{2}$. It is not hard to see that if buyer 1 starts with a bid $s_{2}$ with seller 1 (instead of $b_{1}$ ), then everything works out the same way, except that when buyer 2 bids something between $s_{2}$ and $b_{1}$, buyer 1 is displaced as high bidder and trades at price $s_{2}$ with seller 2. Deviating to the price $s_{2}$ ensures that buyer 1 will never have to pay a price above $s_{2}$. This strictly improves upon the bid $b_{2}$ provided buyer 1 believes that buyer 2 has a valuation between $s_{2}$ and $b_{1}$ with strictly positive probability. 


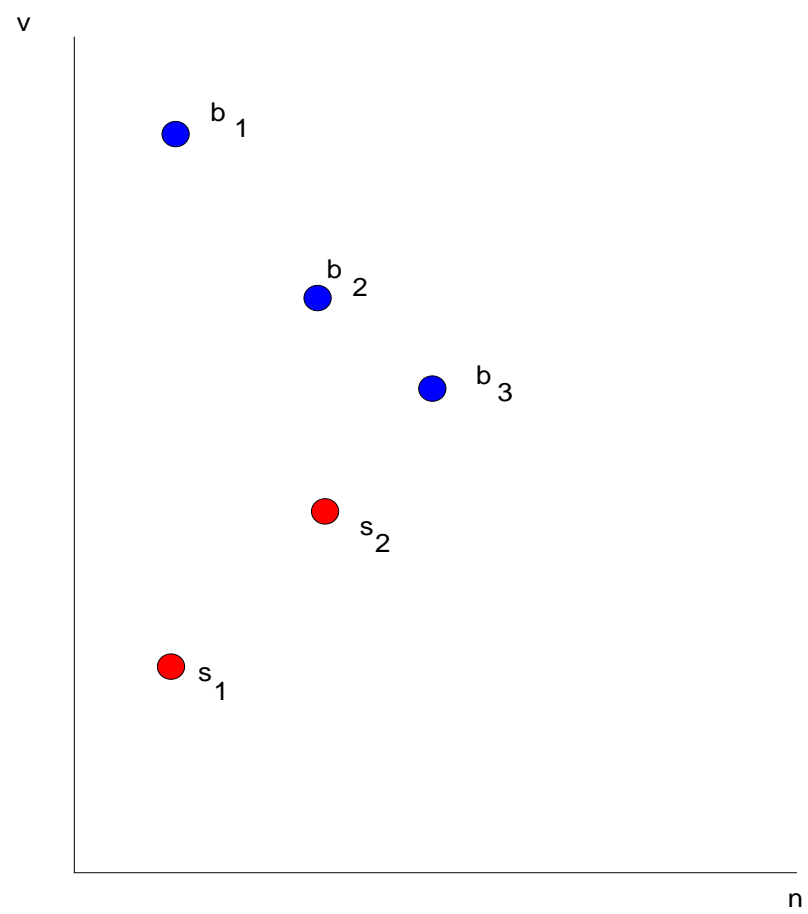

Figure 1:

\section{Efficient Bidding}

The advantage of sequential bidding is that buyers whose valuations are high, but who had the bad luck of bidding against another buyer with an even higher bid, have an option to bid again elsewhere. Unfortunately, this option is not sufficient to guarantee that, conditional on seller's reserve prices, the efficient trades are carried out. The following example demonstrates this.

In Figure 2, given sellers' reserve prices $s_{1}$ and $s_{2}$ the efficient outcome is for buyer 1 to trade with seller 1 . However, consider the following strategies. If a buyer finds that no other bids have been submitted and his valuation is at least $s_{1}$, then he submits a bid equal to $s_{2}$ with seller 1 . If there is a bid at seller 1 , then the buyer bids his valuation with seller 2 provided his valuation is at least as large as $s_{2}$, and refrains from bidding otherwise. This strategy is optimal for the buyer who enters first because she ends up trading with seller 1 at price $s_{1}$. Following this strategy is also optimal for the buyer who enter last, because he believes (correctly) that the buyer who has already entered has bid $s_{2}$ at seller 


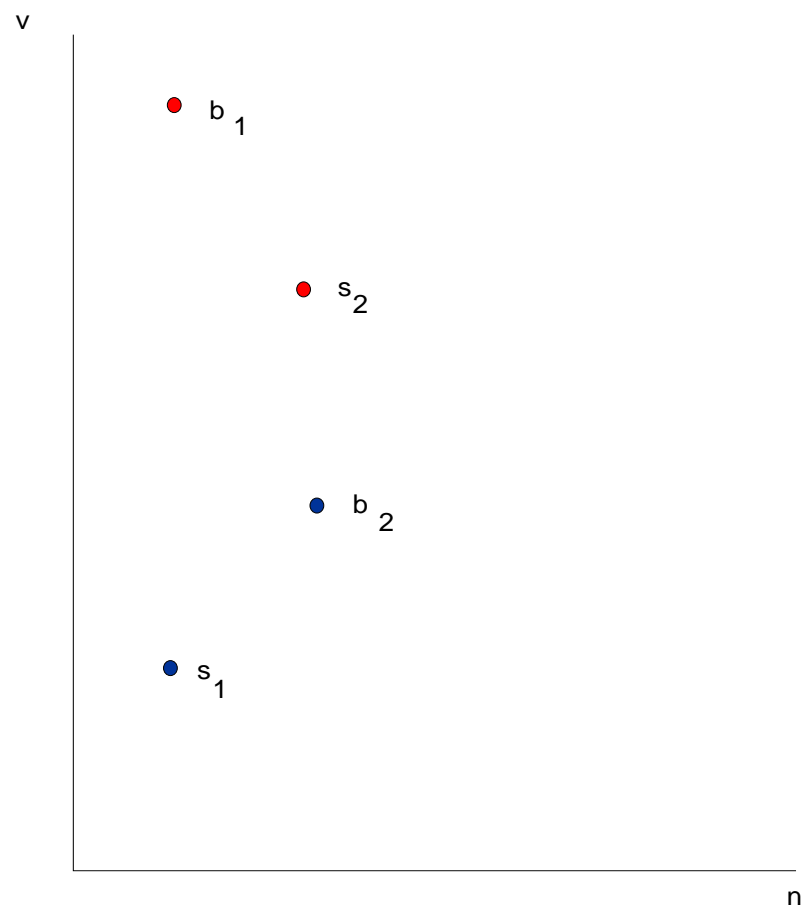

Figure 2:

1. Therefore, there is an equilibrium in which both buyers use this strategy. Given the data in Figure 2, if buyer 2 enters first then in this equilibrium he will trade with seller 1 , and then buyer 1 with a higher valuation will trade with seller 2. Thus, equilibrium outcome of the bidders' game could be inefficient.

It is easy to demonstrate that in this example, as well as in many other ones, the dynamic bidding game has multiple equilibria. We will not attempt to characterize all of them. Rather, our objective is to try to identify bidding equilibria that have nice properties, especially, the ones that are efficient conditional on the announced reserve prices.

To begin, let $\mathbf{b}=\left\{b_{1}, \ldots b_{m}\right\}$ be the vector of buyers' valuations, and let $\mathbf{s}=\left\{s_{1}, \ldots s_{n}\right\}$ be the vector of sellers' reserve prices. Without loss of generality, assume that sellers are indexed in such a way that $s_{1} \leq s_{2} \cdots \leq s_{n}$, while buyers are indexed the opposite way so that $b_{1} \geq b_{2} \cdots \geq b_{3}$. Following Satterthwaite and Williams (1089), let $\mathbf{v}=\left\{v_{1}, v_{2}, \ldots v_{m+n}\right\}$ be vector with entry $v_{i}$ equal to $i$-th lowest value among buyers' valuations and sellers' reserve prices. If sellers' reserve prices were equal to their true valuations, then the efficient set of trades 
occurs if buyers whose valuations exceed $v_{m}$ trade with sellers whose reserve prices are equal or less than $v_{m}$. To see why, note that initially there are $n$ sellers holding one unit of output and $m$ buyers. Therefore, after all trades are completed exactly $n$ traders will own the good and the other $m$ will not. Efficiency requires that the traders who end up without the good have the lowest valuations or costs.

We now define the symmetric strategy $\sigma^{*}$ by specifying how each buyer should bid when his (her) turn comes. To help describe the strategy, make the natural definition and say that a bid is successful if the bidder who makes it becomes high bidder, and that the bid is unsuccessful otherwise.

Definition 1 The symmetric strategy $\sigma^{*}$ is defined as follows: if it is the buyer's turn to bid then

(a) if the buyer is the current high bidder at any auction, or if the buyer's valuation is less than or equal to the lowest standing bid, the buyer should pass;

(b) otherwise, if there is a unique lowest standing bid, the buyer should submit a bid with the seller offering this lowest standing bid. The bid should be equal to the lowest value on the grid that exceeds this low standing bid;

(c) otherwise, if more than one seller has the lowest standing bid, the buyer should submit the same bid as in (b) with equal probability at each such seller where either the seller has not recevied a bid, or the last bid the seller received was unsuccessful. If the last bid was successful with all sellers holding the lowest standing bid, then the buyer should bid with each of them with equal probability.

Before proceeding, it might help to visualize the path generated when buyers use strategy $\sigma^{*}$ in a simple demand-supply style diagram. We refer again to Figure 1, where the valuations and reserve prices of three buyers and two sellers are shown. This is simply an example designed to illustrate the way the strategies work, so for convenience we can assume that the grid of feasible bids coincides with the set of sellers' reserve prices $s_{1}, s_{2}$ and buyers' valuations $b_{3}, b_{2}, b_{1}$. Assume further than buyers enter in reverse order of their valuations, so buyer 3 with valuation $b_{3}$ enters first, then buyer 2 then buyer 1 . According to strategy $\sigma^{*}$, when buyer 3 enters he bids $s_{2}$ with seller 1 because seller 1 initially has the lowest standing bid and $s_{2}$ is the lowest valuation on the grid that exceeds $s_{1}$. This bid will be successful but it will have no effect on seller 1 's standing bid. Buyer 2 will bid the same amount with seller 1, since seller 1 's standing bid still has the lowest standing bid.

The bid by Buyer 2 is unsuccessful, but it makes the standing bid with seller 1 rise to $s_{2}$. Observe that unsuccessful bids will always change a seller's standing bid, since buyers must submit bids above the current standing bid. As buyer 3 was the first to submit this bid, he remains the high bidder and passes by (a), so buyer 2 has the chance to submit a new bid. Now both sellers have the same 
standing bid. The last bid with seller 1 was not successful, and seller 2 has not yet received a bid. The lowest value that exceeds the standing bid $s_{2}$ is $b_{3}$, so by (c) buyer 2 will bid $b_{3}$ with equal probability at seller 1 (case (a)) or seller 2 (case (b)). In either case, buyer 2's bid will be successful and it will not affect either of the standing bids. Note that a successful bid may or may not change a seller's standing bid.

Consider case (a) first. Since buyer 2 displaces buyer 3, buyer 3 immediately submits a bid equal to $b_{3}$ with seller 2 , since seller 2 has yet to receive a bid in this case. The bid is successful, and again, this bid does not affect either of the standing bids which remain equal to $s_{2}$. When buyer 1 enters he finds that the last bid with both sellers was successful, so he will randomize. Whichever sequence he chooses, the bid $b_{3}$ will be unsuccessful and will raise the standing bids at both sellers will rise to $b_{3}$. Then buyer 1 will bid $b_{2}$ choosing randomly between the two sellers (since the last bid was unsuccessful at both locations). If buyer 1 bids first with seller 1 , then he will displace buyer 2 , who will, in turn, bid $b_{2}$ at seller 2 and become a winning bidder there. Bidding will then stop, and buyers 1 and 2 will trade at price $b_{3}$. If he bids first with seller 2 , his bid will be successful and all bidding will stop.

Now consider case (b). When buyer 1 enters, he will submit bid $b_{3}$ at seller 1 and displace bidder 3 without raising the reserve price above $s_{2}$. Buyer 3 will then submit bid $b_{3}$ at sellers 1 and 2 in random order, which will raise the standing bid at both sellers to $b_{3}$, but bidder 3 will still not be a winning bidder. The bidding will then stop and buyer 1 and 2 will trade.

This example conveys the essential idea. Buyers bid up prices with each seller as slowly as possible. For this reason, high valuation buyers are never trapped into paying higher prices if another high-valuation buyer accidentally bid against them. In this example, the efficient trades occur and both sellers trade at a uniform price equal to buyer 3's valuation. The randomness on the equilibrium path makes possible different profiles of winning bids and different pairwise matching between buyers and sellers who trade. However, the uniform trading price is uniquely determined by the profiles of buyers' valuations and sellers' reserve prices. These properties of the strategy rule $\sigma^{*}$ are quite general, as the following theorem demonstrates.

Theorem 2 The outcome in which all buyers use the strategy $\sigma^{*}$ is a perfect Bayesian equilibrium. For each array of valuations and reserve prices $\mathbf{v}$, each buyer whose valuation is above $v_{m}$ trades with some seller whose reserve price is no larger than $v_{m}$. All trades occur at the price $v_{m}$.

The proof of this and the other main theorems in the paper is included in the appendix.

According to strategy $\sigma^{*}$, all active buyers focus their bids on seller(s) with the lowest standing bids irrespective of buyers' valuations, beliefs and the standing bids. The active bidders continue to bid up the standing bid with this group of sellers (which could consist of only one seller) until their standing bids reach 
the level of the standing bids or reserve prices at the next group of sellers, i.e. the second lowest level. Then bidding continues with sellers from both groups until the lowest standing bid reaches the level of reserve price or standing bid at the next group of sellers, and so on. This process continues until all remaining active bidders become high bidders with different sellers.

It is notable that strategy $\sigma^{*}$ contains the same set of rules on the equilibrium path as well as off the equilibrium path. But, although $\sigma^{*}$ generates a relatively simple path, the proof that playing $\sigma^{*}$ constitutes an equilibrium is not straightforward because the dynamic bidding game is sufficiently complex.

We provide the proof by showing that a buyer who at any stage of the bidding game submits a higher bid than what is prescribed by $\sigma^{*}$, or who bids above his valuation can at best raise the trading price. At the same time, a buyer who bids as if his valuation is lower than what it is in reality, can sometimes lower the trading price, but only by giving up a desirable trading opportunity.

This line of argument is similar in spirit to the one that is used to show that bidding the true valuation is an equilibrium in a standard static second price auction. But the analogy is only approximate. ${ }^{11}$ A substantially more complex argument is needed because of the dynamic nature of the bidding process and presence of multiple auctions. To compute the payoff that a buyer gets by following $\sigma^{*}$ from the outset of the game, as well as the payoffs that he could get by deviating, we need to consider arbitrary information sets and characterize the outcomes that occur after the play of the game has reached them. In particular, we need to show that playing $\sigma^{*}$ remains optimal for a buyer no matter what information set is reached, if in the continuation all other buyers follow $\sigma^{*}$.

An important property of the equilibrium where buyers use $\sigma^{*}$ is absence of price dispersion in the final outcome: all trades are executed at the same price. Rule (c) in $\sigma^{*}$ plays the crucial role in achieving this. This rule describes buyers' behavior when there are several sellers with the lowest standing bid, and so the buyer faces the uncertainty regarding the winning bids at these sellers.

The essence of rule (c) is that it allows the buyer to identify which of the sellers with the same standing bid have lower winning bids. Bidding only at such sellers is optimal for the buyer, because then with some probability the buyer will trade at a lower price. At the same time, such bidding ensures that standing bids rise uniformly and eliminates the possibility of price dispersion.

Rule (c) tells the buyer to focus on such sellers with lowest standing bids where there had been less action at the current standing bid, or more precisely, where the last submitted bid was unsuccessful in the sense that the bidder who submitted it did not succeed in displacing the high bidder at the auction. On the path generate by our strategies, a successful bid will be strictly above the standing bid and will displace the lead bidder without changing the standing bid. An unsuccessful bid will raise the standing bid without displacing the winner. Thus, when two standing bids are the same, the one where the last bid was successful will have a larger high bid.

\footnotetext{
${ }^{11}$ As shown above, bidding one's valuation is not an optimal strategy in the dynamic bidding game.
} 
For each array $\mathbf{v}$ of buyers' valuations and sellers' reserve prices, theorem 2 uniquely specifies the price at which all trades will be completed. Precisely, the trading price will be equal to $v_{m}$ which is either the highest valuation among buyers whose bids are unsuccessful, or the highest reservation price among sellers who trade, depending on the actual array of valuations and reserve prices. At the same time, the randomization on the equilibrium path (buyers randomize between the sellers among whom they are indifferent) implies that certain aspects of the outcome will also be random. First, the final matching between sellers and buyers who trade is going to be random. Second, if there are $k>1$ traders whose valuations or costs are equal to $v_{m}$, then any number of them between zero to $k-1$ may trade in equilibrium.

To better understand whether buyers and sellers with valuations and costs equal to $v_{m}$ trade, let us divide the set of buyers into three groups. For any array $\mathbf{v}$, let $M_{1}$ be the set of buyers whose valuations are strictly lower than $v_{m}, M_{2}$ be the set of buyers who have valuations exactly equal to $v_{m}$, while $M_{3}$ be the set of buyers whose valuations are strictly higher than $v_{m}$. Similarly, let $N_{1}, N_{2}$, and $N_{3}$ be the sets of sellers who set their reserve prices below, equal to and above $v_{m}$ respectively. Let $m_{i}\left(n_{i}\right)$ be the number of buyers (sellers) in the set $M_{i}\left(N_{i}\right)$. Theorem 2 says that buyers in $M_{3}$ will surely trade, and that buyers in $M_{1}$ and sellers from $N_{3}$ will not trade.

Corollary 3 Let $v_{m}$ be the $m$-th lowest element in the array $\mathbf{v}$ of buyers' valuations and sellers' reserve prices. If all buyers use the strategy $\sigma^{*}$ in the bidding game, a seller who sets reserve price $s$ s.t. $s<v_{m}$ trades for sure. The number of sellers with reserve price equal to $v_{m}$ who trade trade is between

$$
\max \left[0, m_{3}-n_{1}\right] \quad \text { and } \min \left[n_{2}, m_{3}-\min \left\{0, n_{1}-m_{2}\right\}\right]
$$

The number of buyers with valuation equal to $v_{m}$ who trade is between:

$$
\max \left[0, n_{1}-m_{3}\right] \quad \text { and } \min \left[m_{2}, n_{1}-\min \left\{0, m_{3}-n_{2}\right\}\right]
$$

Proof: see the appendix.

Since all sellers from $N_{2}$ (buyers from $M_{2}$ ) are identical, they have the same chances of trading. Therefore the probability that a seller from $N_{2}$ (buyer from $M_{2}$ trades lies in the interval with boundaries that are derived by dividing the corresponding boundaries on the number of sellers from $N_{2}$ (buyers from $M_{2}$ ) who trade over $N_{2}\left(M_{2}\right) .{ }^{12}$

\footnotetext{
${ }^{12}$ By modifying the definition of strategy $\sigma^{*}$ appropriately, we can support equilibria in which the number of sellers from $N_{2}$ who trade is equal to the lower or upper bound established in corollary 3. To obtain the lower bound, modify $\sigma^{*}$ in the following way. When a buyer faces the choice between several sellers who have the lowest standing bid and who are equivalent with respect to rule (c), he bids at first with those sellers whose original reserve prices were lower than their current standing bid. Then the buyers in $M_{3}$ will submit bids with sellers in $N_{1}$ before they will consider the sellers in $N_{2}$. When the opposite modification on the strategy $\sigma^{*}$ is imposed, we can support an equilibrium that reaches the upper bound on the number of sellers from $N_{2}$ who trade.
} 
The central implications of the corollary 3 is that the outcome of the bidding game is efficient: traders who are left without the good at the end of the day are the ones who have the lower valuations and costs. Note that no matter how many sellers from $N_{2}$ and buyers from $M_{2}$ trade, this has no effect on the other traders and the efficiency of the outcome.

We view the presence of randomness in the final outcome as a strength of our model. In large decentralized markets it is unrealistic to expect all aspects of the bidding process to be entirely deterministic. Yet, we demonstrate that despite the presence of randomness on the equilibrium path, the final price will be uniform and independent of the actual path of bidding.

It is also worth pointing out the connection between our result and equilibrium in the double auction market. Consider a double auction with uniform trading price set equal to $\mathrm{m}^{-{ }^{t h}}$ lowest value among buyers' bids and sellers' asks. Satterthwaite and Williams (1089) refer to it as 'seller's bid double auction'. They show that buyers would bid their true valuations in it and therefore, conditional on the seller's ask prices (reserves), the trading price will be equal to $v_{m}$. Bidding his valuations is optimal for a buyer because his bid can only affect the trading price if the buyer fails to trade. The usual second price logic then implies then applies. Thus, the dynamic bidding game studied here has an equilibrium that generates an outcome identical to the outcome in a seller's bid double auction when sellers' reserves prices are the same set in both markets.

Concluding the discussion of the bidding game, note that neither the description of strategy $\sigma^{*}$ nor the proof that $\sigma^{*}$ is a best reply depends in any way on the distribution of valuations, or the number of buyers and sellers in an auction. ${ }^{13}$ For example, if there is only a single seller, the trade will occur at the reserve price if there is only a single buyer whose valuation is above the reserve, and at the second highest valuation otherwise. This is the same as the outcome in a second price auction. Playing strategy $\sigma^{*}$ remains an equilibrium when there is a large number of sellers. On the other hand, as in the seller's bid double auction, a seller's behavior in our mechanism will typically depend on her beliefs and the number of traders. We turn to the this issue in the next section.

\section{Sellers' Strategies and the Efficiency Result}

The results of the previous section indicate that buyers' equilibrium strategies guarantee that the efficient set of trades occurs when sellers set reserve prices equal to their true valuations. Buyers behavior is efficient because buyers affect the trading price only when they forego attractive trading opportunities.

At the same time, the outcome will not necessarily be efficient if sellers

\footnotetext{
${ }^{13}$ This statement has to be qualified slightly since we impose restrictions on beliefs off the equilibrium path, for example to ensure that no buyer believes that the high bidder in a any auction has a valuation below the standing bid in that auction. This restriction may seem unreasonable if valuations are correlated and prior beliefs conditional on the buyer's own valuation are inconsistent with this.
} 
set reserve prices that are different from their true costs. So we turn to an examination of sellers' behavior in this section.

It may not be optimal for a seller to set a reserve price equal to her true valuation, because her reserve price may affect the trading price in some cases. For example, consider the situation depicted in Figure 1 without buyer 3 (the one with the lowest valuation). From the Figure it is clear that, if buyers use strategy $\sigma^{*}$, the uniform trading price will be equal to the higher reserve price as long as it is below $b_{2}$. Sellers, of course, do not know $b_{2}$. Thus, seller 2 could raise the trading price with a strictly positive probability by raising his reservation price above $s_{2}$. Clearly, the cost of increasing her reserve price to seller 2 is that she would fail to trade if either buyer has valuation between $s_{2}$ and her new higher reserve price.

This tradeoff is similar to the one which traders face in a standard double auction. The rate of convergence of the optimal reserve prices to their true costs in a double auction has been analyzed by Satterthwaite and Williams (1089) in the case where the costs and valuations are independently and continuously distributed over an interval. In the analysis below, we will also maintain the independence assumptions. However we consider a finite grid of valuations which allows us to get a somewhat tighter result.

Thus, assume that buyers' valuations and sellers' costs are distributed independently. Let $f(p) \equiv F(p)-F(p-1)$ and $g(p) \equiv G(p)-G(p-1)$ denote the probability that a buyer's valuation and a seller's cost respectively are equal to $p$ exactly. Let $\bar{g}=\min _{p \in \mathcal{D}} g(p)$ and $\bar{f}=\min _{p \in \mathcal{D}} f(p)$. We further assume that $\bar{g}>0$ and $\bar{f}>0$.

We consider a sequence of markets that get larger as the number of traders increases. For simplicity, we hold the ratio of the number of buyers to the number of sellers constant at $k>0$ i.e., $m=k n$ where $m$ is the number of buyers and $n$ is the number of sellers.

The main result of this section is the following theorem which establishes that setting a reserve price equal to the true cost constitutes an equilibrium strategy for sellers when the number of traders in the market is sufficiently large.

Theorem 4 Suppose that every seller except seller $z$ sets her reserve price equal to her true cost and buyers follow the strategy $\sigma^{*}$. If $m$ and $n$ are sufficiently large, it is optimal for seller $z$ to set reserve price equal to her true cost.

The proof of this theorem is provided in the appendix. It demonstrates that, provided that the number of traders is sufficiently large, a seller with cost $c$ obtains a higher expected payoff by setting reserve price equal to $p-d$ rather than $p$ for $p>c$. The two expected payoffs are the same if the trading price is either above $p$ or below $p-d$. Thus, we need to focus on the situations where seller $j$ 's choice between $p$ and $p-d$ affects the trading price, and where the trading price is equal to $p-d$ or $p$ irrespective of seller $j$ 's choice. The latter situation occurs if several other sellers post the reserve price equal to the trading price. The probability of this event is zero when the costs are 
distributed continuously. Yet, with a discrete set of valuations it occurs with a positive probability and has an important effect. When several sellers are tied at the trading price, a seller may fail to trade even if her reserve price is equal to the trading price, because of the competition from the other sellers posting this reserve price.

Precisely, seller $j$ gets different expected payoffs by setting reserve price equal to $p-d$ or $p$ in the following four cases:

1. the trading price is equal to $p-d$ whether the seller sets her reserve price equal to $p-d$ or $p$, and seller $j$ trades after setting price $p-d$.

2 . the trading price is equal to $p-d(p)$ when the seller sets her reserve price equal to $p-d(p)$ and the seller fails to trade at price $p^{14}$.

3. the trading price is equal to $p$ whether the seller sets her reserve price equal to $p-d$ or $p$, and seller $j$ fails to trade after setting reserve price $p$.

4. the trading price is equal to $p-d(p)$ when the seller sets her reserve price equal to $p-d(p)$ and the seller trades at price $p$.

The seller gets a higher expected payoff by setting reserve price equal to $p-d$ if the effects of (1)-(3) outweigh the effect of (4). In the proof, we ignore the effect of (1) and (2) and show that the effect of (3) alone dominates (4). The effects of (1) and (2) are ignored because when $p-d=c$, the net surplus to seller $j$ from trading at price $p-d$ is zero. Comparing (3) and (4), we can reinterpret our findings as follows: a seller posting price $p-d$ has higher chances of trading at price $p$ than a seller posting price $p$. To understand the intuition behind this result, note that the expected number of sellers posting a reserve price equal to the trading price increases as the number of traders grows. Competition from these sellers implies that with a significant probability a seller posting such reserve price fails to trade. Yet, if this seller posts a reserve price that is slightly lower than the trading price, she will trade with probability 1 and obtain a positive surplus.

\section{Conclusion}

At least two remarks are in order about the results of this paper. First, the equilibrium in buyers' bidding game that we describe is not unique. Although the behavior that occurs in our equilibrium is plausible, alternative equilibria exist and do not generally guarantee efficient allocations. Examples in the paper illustrate this. We do not have a complete characterization of all equilibria in the dynamic bidding game, and of sellers' optimal behavior with respect to reserve prices when they believe that buyers are to play a different equilibrium.

Part of the job of an equilibrium in a decentralized market is to coordinate the matching decisions that buyers and sellers make. Coordination problems

\footnotetext{
${ }^{14}$ It is easy to show that seller $j$ will always trade after posting reserve price $p-d$ in this case
} 
almost always have multiple equilibria and having to choose among them seems inevitable. Second-price auctions, for example, possess asymmetric equilibria in which bidders do not bid their true valuations. There are multiple equilibria in centralized mechanisms like double auctions as well.

Taking the multiplicity problem seriously, we do not want to suggest that traders will play the equilibrium described in this paper under all circumstances. At the same time, the equilibrium behavior that we identify has a number of advantages. It is reasonable-looking, i.e. simple, requires very little computation on the part of traders, and is invariant to the form of the distributions from which costs and valuations are drawn. It also implements the efficient allocation. These properties make us believe that this equilibrium has focal nature, and eventually traders will learn to play it.

Of course, our model does not reproduce all details of the bidding behaviour on the ebay, Amazon or other auction sites. These auctions typically possess additional dynamic aspects that we do not consider. For example, sellers enter at random times, as do buyers. Auctions close at different times. Furthermore, bidding is not completely costless on these sites. Roth and Ockenfels (2000) suggest network congestion and unexpected demands by the family as reasons why bidders may not be able to revise a bid as intended. Nonetheless, our model does provide some insight into the workings of these institutions.

As mentioned above, our model gives a very simple explanation for the fact that buyers do not bid their true valuations. Patient revision of bids provides buyers with the signals that they need in a decentralized market to coordinate their bidding behavior. Deviating from this pattern will often force a buyer to trade at a price that is higher than necessary. The empirical implications of our theory are discussed in the introduction. An extension of the logic of our model suggests an explanation for observed flurry of active bidding close to the end dates in the ebay auctions. In our model, all the available trading opportunities are visible at the beginning of the bidding process. On ebay, these opportunities arise at random times. One of the opportunity costs of submitting a bid on ebay arises from the possibility that a new seller will enter at a lower reserve price after the buyer submits his bid. The buyer may then end up trading at a higher price than he needs to. Effective coordination of bidding among buyers then demands that they refrain from starting the bidding as long as possible. Since auctions at ebay have fixed end dates, at some point the probability that new sellers will enter before the current auction ends becomes small, and buyers will start bidding. One of the implications of our theory is that this late bidding on an auction that is ending will induce a flurry of bidding at other auctions for similar goods.

\section{Appendix}

\section{Proof of Theorem 2:}

At first, let us introduce the following notation. Let the state of the game be the array of buyers' valuations, sellers' standing bids together with the iden- 
tities of buyers who have submitted them, the winning bids together with the identities of the winning bidders, the history of the standing bids and winning bids, plus the identity of the buyer who is the next to move, and the order in which buyers will move in the continuation. Typical state of the game will be denoted by $\Gamma$. Note that there is a one-to-one relationship between the nodes in the game and states of the game. Precisely, state of the game is a full description of the situation at the corresponding node in the game. Define public state of the game as the components of the state of the game that are publicly known. Specifically, public state of the game includes the standing bids, the winning bids, the identities of the winning bidders, the history of all these, and the order of moves.

We will say that state of the game $\Gamma$ is regular if every buyer's valuation is at least as large as any high bid that he holds at $\Gamma$. Note that whether the state of the game is regular or not is unobservable.

We will say that bidder $i$ 's position is consistent with $\sigma^{*}$ if each of $i$ 's high bids has the following property: if $i$ is a high bidder with seller $j$ then either (i) $i$ 's bid is one grid point above seller $j$ 's standing bid and no seller has a lower standing bid than does seller $j$; or (ii) $i$ 's high bid is equal to $j$ 's standing bid. It is not hard to see from the definition that on the path generated by $\sigma^{*}$ all states of the game are regular, and that every buyer's position in these information sets will be consistent with $\sigma^{*}$.

If in state $\Gamma$ one or more buyers are high bidders at more than one seller, we will replace the continuation of the true game from the node that corresponds to $\Gamma$ with the one where additional 'phantom' buyers are added to the game, but where each buyer (real or phantom one) has a winning bid at one seller at most. The phantom buyers are added in the following way. If buyer $i$ is a winning bidder at multiple (say, $l>1$ ) sellers, choose any one of $i$ 's highest winning bids and consider that buyer $i$ possesses only this winning bid. For each of $i$ 's $l-1$ other high bids, create a phantom buyer $i^{k}(2 \leq k \leq l-1)$ whose valuation is equal to this winning bid. Then, define $\tilde{\mathbf{v}}$ to be the vector of the valuations of the real and phantom bidders, and the current standing bids in state $\Gamma$. If there are $\tilde{m} \geq m$ buyers and phantom buyers, then $\tilde{\mathbf{v}}$ has dimension $\tilde{m}+n$. Let $\tilde{v}_{r}$ is the $r$-th smallest element of vector $\tilde{\mathbf{v}}$.

Define $B_{\Gamma}$ to be the smallest set of buyers (including phantom buyers) at state $\Gamma$ such that if $B_{\Gamma}$ contains $m_{\Gamma}$ bidders, every buyer who is not in $B_{\Gamma}$ is high bidder with a seller whose standing bid strictly exceeds $\tilde{v}_{m_{\Gamma}}$.

Lemma 5 If state of the game $\Gamma$ is regular, then the set $B_{\Gamma}$ is non-empty and unique. Furthermore if $B_{\Gamma}$ contains $m_{\Gamma}$ buyers, no buyer in $B_{\Gamma}$ is high bidder at a seller with standing bid above $\tilde{v}_{m_{\Gamma}}$.

Proof. Let $B_{0}$ be the set of buyers who are not high bidders at any seller. Suppose that $B_{0}$ includes $\tau_{0}$ buyers. By definition, $B_{0} \subset B_{\Gamma}$. Let $s_{0}$ be the lowest standing bid. If $\tilde{v}_{\tau_{0}}<s_{0}$ then $B_{\Gamma}=B_{0}$, and we are done. Otherwise, $B_{\Gamma}$ must contain all buyers who are high bidders at sellers with standing bid $s_{0}$. To see this, consider any candidate set $B_{1}$ with $\tau_{1} \geq \tau_{0}$ members which contains 
$B_{0}$. Since $\tilde{v}_{\tau_{1}} \geq \tilde{v}_{\tau_{0}} \geq s_{0}$, a high bidder at $s_{0}$ does not satisfy the condition for exclusion from $B_{1}$.

We can now apply the same argument recursively. Let $B_{l}$ be the set that includes all buyers from $B_{0}$ and all buyers who are high bidders at the lowest, second lowest, $\ldots, l$-th lowest standing bids. Suppose that there are $\tau_{l}$ buyers in $B_{l}$, and no subset of $B_{l}$ satisfies the definition of $B_{\Gamma}$. If $\tilde{v}_{\tau_{l}}<s_{l+1}$, where $s_{l+1}$ is the $l+1$-th lowest standing bid, then $B_{\Gamma}=B_{l}$. Otherwise, $B_{\Gamma}$ must contain all buyers who are high bidders with sellers whose standing bids are less than or equal to $s_{l+1}$. Continue in this way until $l^{\prime}$ s.t. $B_{l^{\prime}}$ satisfies the definition of $B_{\Gamma}$ or until all buyers have been included in $B_{\Gamma}$.

It is immediate that no buyer in $B_{\Gamma}$ is a high bidder at a seller whose standing bid exceeds $\tilde{v}_{m_{\Gamma}}$ (where $m_{\Gamma}$ is the number of bidders in $\Gamma$ ), for such a bidder can be excluded from $B_{\Gamma}$ to form a strictly smaller set satisfying the definition.

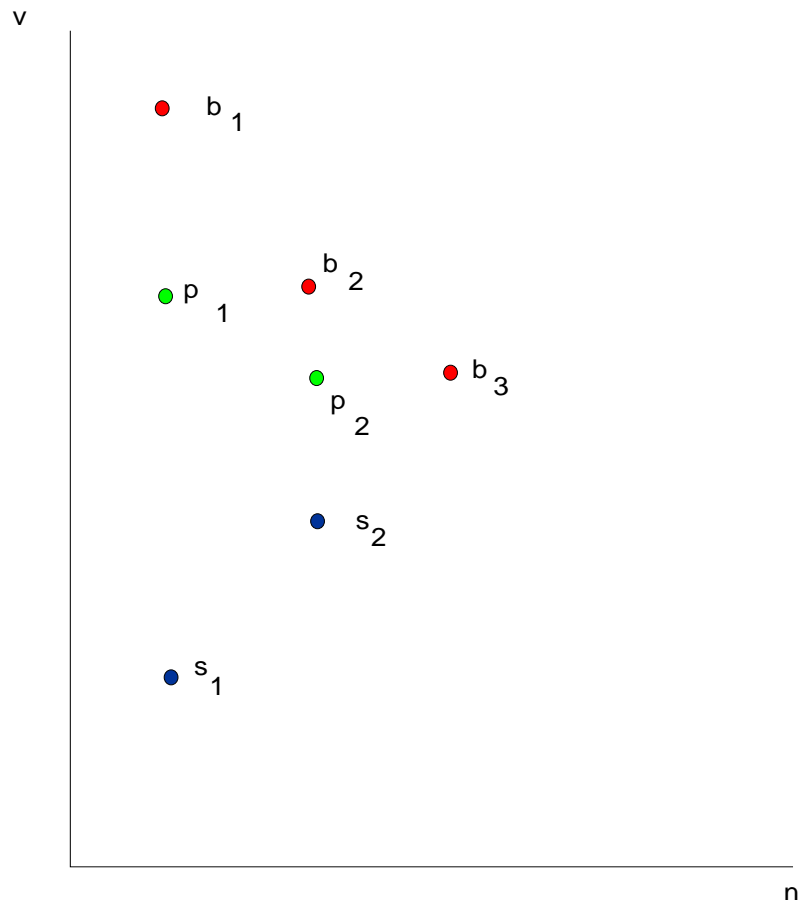

Figure 3:

Figure 3 provides an example of a situation with phantom bidders. Buyers' valuations $b_{1}, b_{2}, b_{3}$ and sellers' reserve prices $s_{1}, s_{2}$ are the same as in Figure 1. 
However, in the state which we are considering buyer 1 has submitted two high bids, $p_{1}$ with seller 1 and $p_{2}=b_{3}$ with seller 2 , but no other buyer has entered yet. The standing bids remain $s_{1}$ at seller 1 and $s_{2}$ at seller 2 . Since buyer 1 is high bidder at two sellers, we generate a phantom buyer whose valuation and bid are both equal to $p_{2}$. There are then 4 buyers including the phantom buyer, instead of only 3 in the original game). The vector $\tilde{\mathbf{v}}$ of valuations and standing bids in increasing order is given by $\left(s_{1}, s_{2}, p_{2}, b_{3}, b_{2}, p_{1}, b_{1}\right) . B_{\Gamma}$ is the whole set of buyers, including the phantom buyer. Consequently, $m_{\Gamma}=4$ and $\tilde{v}_{4}=p_{2}=b_{3}$. None of the buyers is a high bidder with a seller whose standing bid is above $p_{2}$, so this is the right choice for $B_{\Gamma}$.

To understand the outcome on the continuation path where all buyers follow $\sigma^{*}$, assume that buyer 3 enters first. Buyer 3 will bid up the standing bid with each of the two sellers until both standing bids reach his valuation $b_{3}$. Buyer two will then choose between the two sellers randomly. Whichever choice buyer 2 makes, he will trade at price $p_{2}$, while buyer 1 will trade either at price $p_{2}$ or $p_{1}$ depending on where buyer 2 ends up submitting his bid first.

Figure 3 illustrates why $\sigma^{*}$ may not generate uniform prices in the continuation after arbitrary states. It also shows why buyers will want to avoid submitting high bids early, even though each seller runs a second price auction. Buyer 1 may have to pay $p_{1}$ instead of $p_{2}$ if bidder 2 bids at seller 1 first.

To see another example where non-uniform prices can result, suppose that the high bids $p_{1}$ and $p_{2}$ are held by buyers 1 and 2 respectively and eliminate buyer 3 from the data in Figure 3. In this case no buyer is a high bidder at more than one seller, but both buyers use strategies that are inconsistent with $\sigma^{*}$. If they subsequently revert to $\sigma^{*}$ then bidding will stop and buyer 1 will trade at the current standing bid $s_{1}$, and buyer 2 will trade at price $s_{2}$.

Lemma 6 Consider any regular state $\Gamma$ and suppose that all buyers use $\sigma^{*}$ in the continuation. Then no buyer whose position is consistent with $\sigma^{*}$ from the start of the game up to $\Gamma$ will trade at a price above $\tilde{v}_{m_{\Gamma}}$.

Proof. The proof is by contradiction. Thus, suppose that some buyer $i$ from $B_{\Gamma}$ whose position is consistent with $\sigma^{*}$ in all states of the game up to $\Gamma$ ends up trading at price $p>\tilde{v}_{m_{\Gamma}}$ with some seller $j$. We need to consider three cases:

Case (a). When buyer $i$ submits her last bid $b^{i}>\tilde{v}_{m_{\Gamma}}$, the standing bid at seller $j$ is equal to $p$. Since $i$ follows $\sigma^{*}$, the lowest standing bid at this point must be $p$.

Let $k$ be the number of buyers and phantom bidders in $B_{\Gamma}$ whose valuations are greater than $\tilde{v}_{m_{\Gamma}}$. By the definition of $\tilde{v}_{m_{\Gamma}}$, there must be at least $k$ sellers whose standing bids in state $\Gamma$ are $\tilde{v}_{m_{\Gamma}}$ or lower. All these sellers have standing bids of at least $p$ when $i$ submits bid $b^{i}$ at seller $j$. When buyer $i$ submits her bid $b^{i}$, he cannot be a high bidder with some seller, so there are only $k-1$ bidders and phantom bidders who can be high bidders with these $k$ sellers at standing bids of at least $p$. So at least one of the bidders and phantom bidders other than $i$ must be high bidder with more than one seller. Since by construction each 
bidder and phantom bidder is high bidder with at most one seller in state $\Gamma$, some buyer must have submitted multiple high bids above $\tilde{v}_{m_{\Gamma}}$ in the continuation following $\Gamma$. This is inconsistent with part (i) in the definition of $\sigma^{*}$.

Case (b). Buyer $i$ submits his last bid $b^{i}$ in the continuation following state $\Gamma$ when the standing bid $s_{j}$ at seller $j$ is below $p$.

Since $i$ follows $\sigma^{*}, b^{i}=s_{j}+d$. Hence, $b^{i}=p$. As buyer $i$ ends up trading at $p$, bid $b^{i}$ makes him the winning bidder. However, the standing bid cannot change at this point, because if it did, it would imply that $b^{i}>s^{j}+d$.

Since the standing with seller $j$ eventually rises to $p$, some other buyer, say $i^{\prime}$, must submit a bid $p$ with seller $j$ after $i$ has submitted her last bid $b^{i}=p$ and while the standing bid is still $s^{j}$. The identity of the winning bidder at seller $j$ has changed after the standing bid has reached $s^{j}$. Therefore, buyer $i^{\prime}$ will bid at $j$ only if $s^{j}$ is the lowest standing bid and the identity of the winning bidder has changed at all sellers with standing bid $s_{j}$ including sellers whose standing bids are below $\tilde{v}_{m_{\Gamma}}$ in state $\Gamma$. This implies that all $k$ sellers who in state $\Gamma$ have standing bids below $\tilde{v}_{m_{\Gamma}}$ have winning bids above $s^{j} \geq \tilde{v}_{m_{\Gamma}}$.

Since the state $\Gamma$ is regular and since buyer $i^{\prime}$ will not submit bid $p$ if he is already high bidder with some seller, there are only $k-1$ buyers from $B_{\Gamma}$ and phantom bidders who can be high bidders with these $k$ sellers at standing bids of at least $p=s_{j}+d$. So at least one of the bidders and phantom bidders other than $i^{\prime}$ must be high bidder with more than one seller. By construction, each bidder and phantom bidder is high bidder with at most one seller in state $\Gamma$. Therefore, some buyer must have submitted multiple high bids above $\tilde{v}_{m_{\Gamma}}$ in the continuation following $\Gamma$. This is inconsistent with part (i) in the definition of $\sigma^{*}$.

Case (c). At state $\Gamma i$ is holding her final winning bid $b^{i}>\tilde{v}_{t a u}$ at seller $j$ with standing bid $s^{j^{\prime}} \leq \tilde{v}_{m_{\Gamma}}$. Since $i$ is in $B_{\Gamma}$ and his position is consistent with $\sigma^{*}$, we must have $s^{j^{\prime}}=\tilde{v}_{t a u}$ and $p=b^{i}=\tilde{v}_{t a u}+d$. Since $i$ 's position is consistent with $\sigma^{*}$ on the whole path to $\Gamma$, bidder $i$ 's last bid has changed the identity of the winning bidder but not the standing bid.

Since the standing with seller $j$ eventually rises to $p$, some other buyer, say $i^{\prime}$, must submit a bid $p$ with seller $j$ after $i$ has submitted her last bid $b^{i}=p$ and while the standing bid is still $s^{j}$.

Since the standing with seller $j$ eventually rises to $p=\tilde{v}_{t a u}+d$, some other buyer, say $i^{\prime \prime}$, must submit a bid $\tilde{v}_{\text {tau }}$ with seller $j$ in the continuation following state $\Gamma$. To complete the proof, apply the same argument as in (b).

Lemma 7 Let $\Gamma$ be a regular state. If all buyers use $\sigma^{*}$ in the continuation, then no trader will trade at a price below $v_{m_{\Gamma}}$.

Proof. Suppose that some buyer trades at price $p<v_{m_{\Gamma}}$. Then there is at least one seller whose standing bid in state $\Gamma$ is strictly less than $v_{m_{\Gamma}}$. Let the number of such sellers be $r_{1}$. Also, let $B_{1}$ be the set of buyers with valuations equal to or greater than $v_{m_{\Gamma}}$ and who in state $\Gamma$ are either winning bidders with sellers whose standing bids are below $v_{m_{\Gamma}}$ or are not winning bidders at any seller. Denote the number of buyers in $B_{1}$ by $k_{1}$. By definition $B_{1} \subset B_{\Gamma}$. 
First, we will show that $k_{1}>r_{1}$. The proof is by contradiction. Thus, suppose otherwise i.e. $k_{1} \leq r_{1}$. By definition of $v_{m_{\Gamma}}$, there are $k_{2}>r_{1}$ buyers and phantom buyers in $B_{\Gamma}$ whose valuations are $v_{m_{\Gamma}}$ or higher. Therefore, there are $k_{2}-k_{1}>0$ buyers in $B_{\Gamma}$ each of whom has valuations at or above $v_{m_{\Gamma}}$ and is a high bidder with a seller whose standing bid in $\Gamma$ is equal to $v_{m_{\Gamma}}$. Eliminating these buyers from set $B_{\Gamma}$ we obtain a strictly smaller set $B_{\Gamma}^{\prime}$.

Thus, $B_{\Gamma}^{\prime}$ is the union of the set of buyers who have valuations below $v_{m_{\Gamma}}$ (let there be $k_{0}$ of them) and set $B_{1}$. The total number of buyers in $B_{\Gamma}$ is $m_{\Gamma^{\prime}}=k_{0}+K_{1}$. Since $r_{1}>k_{1}, v_{m_{\Gamma^{\prime}}}<v_{m_{\Gamma}}$. Hence, every buyer who is not in $B_{\Gamma^{\prime}}$ is a high bidder with a seller whose standing bid exceeds $v_{m_{\Gamma^{\prime}}}$. This contradicts the fact that $B_{\Gamma}$ is the smallest set that has such property in $\Gamma$.

If trade occurs in the continuation at price $p<v_{m_{\Gamma}}$, then on the continuation path no buyer submits a bid when the standing bid with some seller is $p$. Since $k_{1}>r_{1}$, it follows that in the continuation following $\Gamma$ at least one of the buyers from the set $B$ must has become a high bidder with a seller whose standing bid in $\Gamma$ is $v_{m_{\Gamma}}$ or higher. Submitting such a bid when there is a standing bid below $v_{m_{\Gamma}}$ is inconsistent with $\sigma^{*}$, so at least one buyer has to deviate from $\sigma^{*}$ in order for this outcome to occur. This contradiction proves the result.

Lemma 8 Let $\Gamma$ be any regular state. If all buyers use $\sigma^{*}$ in the continuation, then every buyer who is not in $B_{\Gamma}$ trades with the seller with whom he is high bidder in state $\Gamma$ at this seller's standing bid in $\Gamma$.

Proof. Buyers who are not in $B_{\Gamma}$ are all high bidders with sellers whose standing bids exceed $v_{m_{\Gamma}}$. If they all use $\sigma^{*}$ in the continuation, none of them will be displaced as a higher bidder unless some buyer from $B_{\Gamma}$ submits a bid with one of the sellers whose standing bid is strictly above $v_{m_{\Gamma}}$. Such a bid cannot occur because by Lemma 7 , a buyer from $B_{\Gamma}$ whose valuation is above $v_{m_{\Gamma}}$ and who follows $\sigma^{*}$ is guaranteed to trade if he submits a bid equal to $v_{m_{\Gamma}}+d$ at one of the sellers whose standing bid in state $\Gamma$ does not exceed $v_{m_{\Gamma}}$.

One implication of Lemmas $7-8$ is that any buyer with valuations above $v_{m_{\Gamma}}$ whose initial position is consistent with $\sigma^{*}$ and who follows the strategy $\sigma^{*}$ in the continuation will trade at a price equal to $v_{m_{\Gamma}}$ provided that all other buyers follow $\sigma^{*}$. Consider the implications of this result starting from the initial state $\Gamma_{0}$ in which no buyer has yet submitted a bid. In this state state, all buyers' positions are consistent with $\sigma^{*}$ (trivially since no buyer is a high bidder). Therefore, if all buyers follow $\sigma^{*}$ in the continuation, then all trades will take place at price $v_{m}$.

Lemma 9 Let $\Gamma$ be a regular state, and suppose that every buyer's position is consistent with $\sigma^{*}$. Then in the continuation no buyer can increase his payoff by choosing an action other than that specified by $\sigma^{*}$ provided that the other buyers follow $\sigma^{*}$.

Proof. By lemmas 7 and 6 , if in state $\Gamma$ every buyer's position is consistent with $\sigma^{*}$ and all buyers follow $\sigma^{*}$ then: (i) all trades will take place at price $v_{m_{\Gamma}}$. 
(ii) A buyer $i$ whose valuations strictly exceeds $v_{m_{\Gamma}}$ will trade for sure at this price.

Consider now any continuation path induced by a series of deviations by buyer $i$ when the other buyers follow $\sigma^{*}$. Each such path has finite length since the other bidders will eventually stop bidding and $i$ cannot bid against himself. Thus $i$ must trivially revert to $\sigma^{*}$ at some point along the path (for example when he stops bidding). Since $i$ must always submit bids at least as high as an existing standing bid, the lower bound on $i$ 's trading price given by Lemma 7 along any such path can never be lower than $v_{m_{\Gamma}}$.

Say that buyers' beliefs are $\sigma^{*}$-consistent if each buyer believes that: (i) every other buyer's valuation is drawn from the prior distribution of valuations conditional on each high bidder's valuation being at least as large as the maximum of her high bids; (ii) each of the other buyers' high bids is consistent with $\sigma^{*}$. In other words, buyers' beliefs are $\sigma^{*}$-consistent if every buyer believes that the only states that occur with positive probability are regular states in which all other buyers' positions are consistent with $\sigma^{*}$.

The proof that $\sigma^{*}$ constitutes a Bayesian equilibrium in the bidding game given sellers' announced reserve prices then follows immediately from Lemma 9 since deviations from $\sigma^{*}$ may raise, but can never lower a buyer's trading price in every information set that the buyer thinks occurs with positive probability.

\section{Proof of corollary 3:}

Suppose that seller $j$ posts reservation price $s$ s.t. $s<v_{m}$. According to theorem 2 , when buyers use strategy $\sigma^{*}$ all trades occur at price $v_{m}$. Consider the last bid in the bidding game submitted by some buyer $i^{\prime}$. It must be equal to $v_{m}$ or $v_{m}+d$. If the last bid is equal to $v_{m}+d$, then the lowest standing bid at this point must be $v_{m}$. Hence, seller $j$ will trade.

Suppose now that the last bid in the game is equal to $v_{m}$ and is submitted at some seller $j^{\prime}$. Then before the last bid is submitted the standing bid at $j^{\prime}$ must be equal to $v_{m}-d$, and after the last bid is submitted the standing bid at $j^{\prime}$ must increase to $v_{m}$. Therefore, bidder $i$ is not a winning bidder at the end of the auction. This can happen only if at this point the standing bid at seller $j$ is $v_{m}$. Hence, seller $j$ must trade in this case also.

Consider set of sellers $N_{2}$ who post reserve prices equal to $v_{m}$. A seller from $N_{2}$ trades only if a buyer from $M_{3}$ bids with her. After accounting for sellers from $N_{1}$ who trade for sure, the number of sellers from $M_{3}$ who are available to bid with sellers from $N_{2}$ is at least $m_{3}-n_{1}$. This gives the lower bound on the number of sellers from $N_{2}$ who trade.

To obtain the upper bound, note that on the equilibrium path buyers from $M_{2}$ will bid only with sellers from $N_{1}$ while the standing bids at these sellers are below $v_{m}$. Consider the first time $t$ when the lowest standing bid in the market reaches $v_{m}$. With a positive probability, the realizations of random order of bidding and the randomizations by buyers between the sellers among whom they are indifferent is such that at time $t \mathrm{~m}^{\prime}$ buyers from $M_{2}$ are the high bidders at sellers from $N_{1}$, where $m^{\prime}$ is between $\max \left\{0, n_{1}-m_{3}\right\}$ and 
$\min \left\{m_{2}, n_{1}\right\}$. Also, with a positive probability all buyers from $M_{3}$ who at time $t$ are not winners yet, will bid at sellers from $N_{2}$ first. The number of buyers from $M_{3}$ who will bid in this way is equal to: $m_{3}-\left(n_{1}-m^{\prime}\right)$. Substituting for $m^{\prime}$ we get the upper bound on the number of sellers from $N_{2}$ who trade.

The proof establishing the lower and upper bounds on the number of buyers from $M_{2}$ who trade is similar and is, therefore, omitted.

\section{Proof of theorem 4:}

Consider seller $z$ with cost $c$. We will demonstrate that seller $z$ 's expected payoff decreases in her reserve price $p$ if $p>c$. To accomplish this, we will compare the expected payoffs that the seller gets when she posts reserve prices equal to $p$ and $p-d$.

In the previous section we have demonstrated that all trades will be completed at a uniform trading price equal to $v_{m}$-the m-th lowest element in $\mathbf{v}$, the vector of the true buyers' valuations and sellers' reserve prices which, by assumption of the theorem, are equal to the true costs for all sellers except $z$. Thus, the trading price will be equal to $p^{T}$ if and only if the following two necessary and sufficient conditions hold. First, the number of sellers and buyers whose reserve prices and valuations respectively are strictly below $p^{T}$ does not exceed $m-1$. Second, the number of sellers and buyers whose reserve prices and valuations respectively are no greater than $p^{T}$ is at least $m$.

We will use the following notation that has been introduced above: $m_{1} / m_{2} / m_{3}$ is the number of buyers with valuations strictly below $p$ / equal to $p$ / strictly above $p$. Similarly, $n_{1} / n_{3}$ is the number of sellers with costs strictly below $p /$ strictly above $p$. Also, let $n_{2}^{\prime}$ be the number of sellers, other than $z$ with costs equal to $p$. Obviously, $n_{2}^{\prime}=n_{2}-1, m_{1}+m_{2}+m_{3}=m$ and $n_{1}+n_{2}^{\prime}+n_{3}=n-1$.

At first, let us establish the following two claims.

Claim 1. Suppose that if seller $z$ sets reserve price $p$, then the trading price is $p_{T}$ s.t.p $<p_{T}$. Then, if seller $z$ sets a different reserve price $p^{\prime}<p_{T}$, the trading price will also be $p_{T}$. The buyer will trade in both cases.

Proof: The trading price is equal to $v_{m}$ which is not affected by a change in the reserve price $p$ set by $z$ as long as $p<v_{m}$.

Claim 2. Suppose that if seller $z$ sets reserve price $p$, then the trading price is $p_{T}$ s.t. $p>p_{T}$. Then, if seller $z$ sets reserve price $p^{\prime \prime}>p_{T}$ the trading price will also be $p_{T}$. The buyer will fail to trade in both cases.

Proof: The trading price is equal to $v_{m}$ which is not affected by a change in the reserve price $p$ set by $z$ as long as $p>v_{m}$.

Say that price $p$ is pivotal if the trading price is equal to $p$ when seller $z$ sets her reserve price equal to $p$. Claims 1 and 2 imply that seller $z$ 's payoffs from setting reserve price equal to $p-d$ or $p$ may be different only if at least one of these reserve prices is pivotal.

Let $P(\Omega)$ denote the probability of event $\Omega$ and $E(y) E(y \mid \Omega)$ denote the expectation (conditional expectation given event $\Omega$ ) of the random variable $y$. 
Then the following lemma presents sufficient condition for theorem 4 to hold.

Lemma 10 Seller $z$ with cost c gets a higher expected payoff by setting reserve price $p-d$ rather than $p$ for $p>c$ if the following condition holds:

$$
\begin{aligned}
& P(p \text { is pivotal }, p-d \text { is not pivotal, seller posting } p \text { fails to trade }) \geq \\
& P(p \text { is pivotal }, p-d \text { is pivotal, seller posting } p \text { trades })
\end{aligned}
$$

Proof. By Claims 1 and 2, it is sufficient to compare the seller's expected payoffs from setting her reserve price equal to $p-d$ and $p$ when at least one of these prices is pivotal. Let us consider all such cases. Note that a seller may fail to trade when her reserve price is pivotal, because there may be other sellers who post this reservation price. Alternatively, there may be buyers with valuations equal to the pivotal price.

1. If $p$ is pivotal, but $p-d$ is not, then irrespective of seller $z$ 's reserve price, $v_{m}$ and hence the trading price are equal to $p$. This follows because in this case we have: $m_{1}+n_{1}<m-1 \leq m_{1}+n_{1}+m_{2}+n_{2}^{\prime}$. Consequently, if $z$ sets reserve price $p-d$ she trades at price $p$ for sure. If she sets reserve price $p$, she may fail to trade if $m_{1}+n_{1}+m_{2}+n_{2}^{\prime} \geq m$.

2. If $p-d$ is pivotal, but $p$ is not, then irrespective of seller $z$ 's reserve price, $v_{m}$ (and hence the trading price) is equal to $p-d$. This follows because in this case we must have $m_{1}+n_{1} \geq m$. Consequently, if $z$ sets reserve price $p$ she fails to trade. If she sets reserve price $p-d$, she may or may not trade.

3. If both $p-d$ and $p$ are pivotal, we must have: $m_{1}+n_{1}=m-1$. Then if seller $z$ posts reserve price $p-d$ she will trade at this price for sure. If the seller sets reserve price $p$, the trading price will be equal to $p$ but seller $j$ may fail to trade if $m_{2}=n_{2}>0$.

Summing up these effects we conclude that seller $z$ with cost $c<p$ obtains a higher payoff by setting reserve price $p-d$ rather than $p$ if and only if the following inequality holds:

$(p-c) P(p$ is pivotal, $p-d$ is not pivotal, seller posting $p$ fails to trade $)+$ $(p-d-c) P(p-d$ is pivotal, $p$ is not pivotal, seller posting $p-d$ trades $)+$ $(p-d-c) P(p-d$ is pivotal, $p$ is pivotal $) \geq$ $(p-c) P(p$ is pivotal, $p-d$ is pivotal, seller posting $p$ trades $)$

Obviously, (2) holds when (1) holds, and these two inequalities are equivalent when $c=p-d$. 
Lemma 11 Inequality (1) holds if:

$$
\begin{aligned}
& E\left(\frac{1+n_{2}^{\prime}+n_{1}+m_{1}-m}{n_{2}^{\prime}+1} \mid m_{1}+n_{1}<m-1 \leq m_{1}+n_{1}+m_{2}+n_{2}^{\prime}\right) \times \\
& \times P\left(m_{1}+n_{1}<m-1 \leq m_{1}+n_{1}+m_{2}+n_{2}^{\prime}\right) \\
& \geq E\left(\frac{1}{n_{2}^{\prime}+1} \mid m_{1}+n_{1}=m-1\right) P\left(m_{1}+n_{1}=m-1\right)
\end{aligned}
$$

Proof. First of all, $p$ is pivotal and $p-d$ is not pivotal if and only if $m_{1}+n_{1}<m-1 \leq m_{1}+n_{1}+n_{2}^{\prime}+m_{2}$. Also, $p$ and $p-d$ are both pivotal if and only if $m_{1}+n_{1}=m-1$.

Next, let us compute the upper bound on the probability with which seller $z$ posting pivotal price $p$ trades conditional on the number of buyers and sellers in each category. By rule (a) of $\sigma^{*}$, seller $z$ can trade only with one of the $m_{3}$ buyers whose valuations are above $p$. By corollary $3, n_{1}$ sellers who post reserve prices below $p$ trade for sure. Some of these $n_{1}$ sellers may trade with buyers whose valuations are equal to $p$. Therefore, the number of buyers who trade with sellers posting $p$ is at most $m_{3}+\min \left\{0, m_{2}-n_{1}\right\}$, and will be lower if some buyers with valuations equal to $p$ do not trade and some buyers with valuations above $p$ trade with sellers whose reserve prices are below $p$.

Seller $z$ competes with the other $n_{2}^{\prime}$ sellers who post reserve price $p$. Since all buyers follow strategy $\sigma^{*}$, a buyer who chooses among such sellers will randomize between them with equal probability. Therefore, the conditional probability that seller $z$ trades is at most

$$
P^{t} \equiv \min \left\{1, \frac{m_{3}+\min \left\{0, m_{2}-n_{1}\right\}}{n_{2}^{\prime}+1}\right\}
$$

Correspondingly, the lower bound on the probability that seller $z$ fails to trade conditional on the number of buyers and sellers in each category is

$$
P^{f} \equiv \max \left\{0, \frac{n_{2}^{\prime}+1-m_{3}-\min \left\{0, m_{2}-n_{1}\right\}}{n_{2}^{\prime}+1}\right\}
$$

Finally, taking expectation of $P^{f}$ conditional on the event that both $p-d$ and $p$ are pivotal, which is equivalent to $m_{1}+n_{1}<m-1 \leq m_{1}+n_{1}+m_{2}+n_{2}^{\prime}$, we obtain that the left-hand side of (1) is at least as large as the left-hand side of (3). Taking expectation of $P^{t}$ conditional on $m_{1}+n_{1}=m-1$ and simplifying we obtain that the right-hand side of (1) is no greater than the right-hand side of (3).

To complete the proof of the theorem it remains to show that (3) holds when $m$ and $n$ are sufficiently large. First of all, note that the right-hand side of (3) is equal to $A_{1}(\alpha)+A_{2}(\alpha)$ where

$$
A_{1}(\alpha) \equiv
$$




$$
\sum_{\hat{m}_{1}=[\alpha m]+1}^{m-1} E\left(\frac{1}{n_{2}^{\prime}+1} \mid n_{1}=m-1-\hat{m}_{1}\right) P\left(m_{1}=\hat{m}_{1}\right) P\left(n_{1}=m-1-\hat{m}_{1}\right)
$$

and

$$
A_{2}(\alpha) \equiv
$$

$\sum_{\hat{m}_{1}=\max \{0, m-n\}}^{[\alpha m]} E\left(\frac{1}{n_{2}^{\prime}+1} \mid n_{1}=m-1-\hat{m}_{1}\right) P\left(m_{1}=\hat{m}_{1}\right) P\left(n_{1}=m-1-\hat{m}_{1}\right)$

where $\alpha \in[0,1]$ and $[\alpha m]$ is defined as the largest integer not exceeding $\alpha m$.

The expression on the left-hand side of (3) is greater than $B_{1}+B_{2}$ where $B_{1}$ and $B_{2}$ are defined as follows:

$$
\begin{gathered}
B_{1} \equiv \\
\sum_{\hat{m}_{1}=\max \{0, m-n\}}^{m-2} E\left(\frac{1+n_{2}^{\prime}+n_{1}+m_{1}-m}{n_{2}^{\prime}+1} \mid m_{1}=\hat{m}_{1}+1, n_{1}=m-\hat{m}_{1}-3\right) \times \\
\times P\left(m_{1}=\hat{m}_{1}+1\right) P\left(n_{1}=m-\hat{m}_{1}-3\right)
\end{gathered}
$$

and

$$
\begin{gathered}
B_{2} \equiv \\
\sum_{\hat{m}_{1}=\max \{2, m-n+4\}}^{m-1} E\left(\frac{1+n_{2}^{\prime}+n_{1}+m_{1}-m}{n_{2}^{\prime}+1} \mid m_{1}=\hat{m}_{1}-2, n_{1}=m-\hat{m}_{1}-1, n_{2}^{\prime} \geq 4\right) \times \\
\times P\left(m_{1}=\hat{m}_{1}-2\right) P\left(n_{1}=m-\hat{m}_{1}-1, n_{2}^{\prime} \geq 4\right)
\end{gathered}
$$

To complete the proof of the theorem, let us prove the following lemma:

Lemma $12 \exists \alpha \in(0,1)$ s.t. if and $m$ (and hence $n$ ) is large enough then $A_{1}(\alpha)<B_{1}$ and $A_{2}(\alpha)<B_{2}$.

Proof. The following expressions are used extensively in the sequel. Let $a+b+c=n-1$ and $r+s+t=m$. Then we have:

$$
\begin{gathered}
P\left(n_{1}=a, n_{2}=b, n_{3}=c\right)=\frac{(n-1) !}{a ! b ! c !} G(p-1)^{a} g(p)^{b}(1-G(p))^{c} \\
P\left(m_{1}=r, m_{2}=s, m_{3}=t\right)=\frac{m !}{r ! s ! t !} F(p-1)^{r} f(p)^{s}(1-F(p))^{t}
\end{gathered}
$$


Part 1: $B_{1}>A_{1}(\alpha)$.

Step 1. Using (5), we have for $2 \leq \hat{m}_{1} \leq m-1$ :

$$
P\left(m_{1}=\hat{m}_{1}-2\right)=\frac{(1-F(p-d))^{2}}{(F(p-d))^{2}} \frac{\hat{m}_{1}\left(\hat{m}_{1}-1\right)}{\left(m-\hat{m}_{1}\right)(m-\hat{m}+1)} P\left(m_{1}=\hat{m}_{1}\right)
$$

Since $1-F(p-d)>\bar{f}>0, \exists m^{\prime}$ and $\alpha_{1}>0$ s.t. if $\hat{m}_{1}>\alpha_{1} m^{\prime}, P\left(m_{1}=\right.$ $\left.\hat{m}_{1}-2\right)>P\left(m_{1}=\hat{m}_{1}\right)$.

Step 2. When $n_{1}=m-\hat{m}-1$, then $n_{2}^{\prime}+n_{3}=\hat{m}_{1}+n-m=\hat{m}_{1}-\frac{k-1}{k} m$. Let $\alpha=\max \left\{\alpha_{1}, \frac{k-1 / 2}{k}\right\}$. (Note that $\alpha<1$.) In this case, $\hat{m}_{1} \geq \alpha m$ implies that $n_{2}+n_{3} \geq \frac{m}{2 k}$. Since $g(p) \geq \bar{g}>0$, the law of large numbers implies that there is $m^{\prime \prime}$ s.t. if $m>m^{\prime \prime}$ then $P\left(n_{2}^{\prime} \geq 4 \mid n_{2}^{\prime}+n_{3} \geq \frac{m}{2 k}\right)>5 / 6$, and hence $P\left(n_{1}=m-\hat{m}_{1}-1, n_{2}^{\prime} \geq 4\right)>\frac{5}{6} P\left(n_{1}=m-\hat{m}_{1}-1\right)$. Therefore,

$$
\begin{gathered}
E\left(\frac{1}{n_{2}^{\prime}+1} \mid n_{1}=m-\hat{m}_{1}-1\right) P\left(n_{1}=m-\hat{m}_{1}-1\right)= \\
E\left(\frac{1}{n_{2}^{\prime}+1} \mid n_{2}^{\prime}<4, n_{1}=m-\hat{m}_{1}-1\right) P\left(n_{2}^{\prime}<4, n_{1}=m-\hat{m}_{1}-1\right)+ \\
E\left(\frac{1}{n_{2}^{\prime}+1} \mid n_{2}^{\prime} \geq 4, n_{1}=m-\hat{m}_{1}-1\right) P\left(n_{2}^{\prime} \geq 4, n_{1}=m-\hat{m}_{1}-1\right) \\
<(1 \times 1 / 6+1 / 5 \times 5 / 6) P\left(n_{1}=m-\hat{m}_{1}-1\right)=1 / 3 P\left(n_{1}=m-\hat{m}_{1}-1\right)
\end{gathered}
$$

On the other hand, we have:

$$
\begin{gathered}
E\left(\frac{1+n_{2}^{\prime}+n_{1}+m_{1}-m}{n_{2}^{\prime}+1} \mid m_{1}=\hat{m}_{1}-2, n_{1}=m-\hat{m}_{1}-1, n_{2}^{\prime} \geq 4\right) \times \\
\times P\left(n_{1}=m-\hat{m}_{1}-1, n_{2}^{\prime} \geq 4\right) \\
\geq \frac{2}{5} P\left(n_{1}=m-\hat{m}_{1}-1, n_{2}^{\prime} \geq 4\right)> \\
\frac{1}{3} P\left(n_{1}=m-\hat{m}_{1}-1\right)
\end{gathered}
$$

Step 3. Choose $m=\max \left\{m_{1}, m_{2}\right\}$. Combining (6), (7) and (8), we conclude that the term corresponding to each $\hat{m}_{1} \geq \alpha m$ in $A_{1}(\alpha)$ is dominated by the corresponding term in $B_{1}$. Therefore, $B_{1}>A_{1}(\alpha)$.

Part 2. $B_{2}>A_{2}(\alpha)$. 
Proof. Consider $\alpha$ defined in part 1. Fix $\hat{m}_{1}$ s.t. $\max \{0, m-n\} \leq \hat{m}_{1} \leq$ $[\alpha m], \hat{n}_{1}=m-\hat{m}_{1}-1$ and $\hat{n}_{2}$ s.t. $0 \leq \hat{n}_{2} \leq n-1-\hat{n}_{1}$. Let us show that the following inequality holds for sufficiently large $m$. (Note that $\hat{n}_{1}>2$ when $m$ is not too small).

$$
\begin{gathered}
E\left(\frac{n_{2}+1+n_{1}+m_{1}-m}{n_{2}+1} \mid n_{1}=\hat{n}_{1}-2, m_{1}=\hat{m}_{1}+1, n_{2}=\hat{n}_{2}+2\right) \times \\
P\left(n_{1}=\hat{n}_{1}-2, m_{1}=\hat{m}_{1}+1, n_{2}=\hat{n}_{2}+2\right)> \\
\frac{1}{n_{2}+1} P\left(n_{1}=\hat{n}_{1}, m_{1}=\hat{m}_{1}, n_{2}=\hat{n}_{2}\right)
\end{gathered}
$$

To establish this, note the following sequence of equalities which holds by computation:

$$
\begin{gathered}
E\left(\frac{n_{2}+1+n_{1}+m_{1}-m}{n_{2}+1} \mid n_{1}=\hat{n}_{1}-2, m_{1}=\hat{m}_{1}+1, n_{2}=\hat{n}_{2}+2\right) \times \\
P\left(n_{1}=\hat{n}_{1}-2, m_{1}=\hat{m}_{1}+1, n_{2}=\hat{n}_{2}+2\right)= \\
\frac{\hat{n}_{2}+1}{\hat{n}_{2}+3} P\left(n_{1}=\hat{n}_{1}-2, m_{1}=\hat{m}_{1}+1, n_{2}=\hat{n}_{2}+2\right)= \\
\frac{\hat{n}_{2}+1}{\hat{n}_{2}+3} P\left(n_{1}=\hat{n}_{1}, m_{1}=\hat{m}_{1}, n_{2}=\hat{n}_{2}\right) \times \\
\frac{g(p)^{2}}{G(p-1)^{2}} \frac{\left(\hat{n}_{1}-1\right) \hat{n}_{1}}{\left(\hat{n}_{2}+2\right)\left(\hat{n}_{2}+1\right)} \frac{F(p-1)}{1-F(p-1)} \frac{m-\hat{m}_{1}}{\hat{m}_{1}+1}= \\
\frac{1}{\hat{n}_{2}+1} P\left(n_{1}=\hat{n}_{1}, m_{1}=\hat{m}_{1}, n_{2}=\hat{n}_{2}\right) \times \\
\frac{g(p)^{2}}{G(p-1)^{2}} \frac{F(p-1)}{1-F(p-1)} \frac{\left(\hat{n}_{2}+1\right)\left(\hat{n}_{1}-1\right) \hat{n}_{1}}{\left(\hat{n}_{2}+2\right)\left(\hat{n}_{2}+3\right)} \frac{m-\hat{m}_{1}}{\hat{m}_{1}+1}
\end{gathered}
$$

Since $\hat{m}_{1} \leq \alpha m$ and $\hat{n}_{1}=m-1-\hat{m}_{1}$, it follows that $m-\hat{m}_{1} \geq(1-\alpha) m$ and $\hat{n}_{1} \geq(1-\alpha) m-1$. Also, $\hat{n}_{2} \leq n-1-\hat{n}_{1}$ implies that $\hat{n}_{2} \leq\left(\frac{1}{k}+\alpha-1\right) m$. Therefore,

$$
\begin{gathered}
\frac{\left(\hat{n}_{2}+1\right)\left(\hat{n}_{1}-1\right) \hat{n}_{1}}{\left(\hat{n}_{2}+2\right)\left(\hat{n}_{2}+3\right)} \frac{m-\hat{m}_{1}}{\hat{m}_{1}+1} \geq \\
\frac{1}{2} \frac{((1-\alpha) m-2)((1-\alpha) m-1)}{\left(\frac{1}{k}+\alpha-1\right) m+3} \frac{(1-\alpha) m}{\alpha m+1}
\end{gathered}
$$

The right-hand side of the above inequality if of the same order as $m$. Therefore, (9) holds when $m$ is sufficiently large (leaving $\alpha$ unchanged).

Finally, note that (9) implies that the term corresponding to each $\hat{m}_{1} \geq \alpha m$ in $A_{2}(\alpha)$ is dominated by the corresponding term in $B_{2}$. Therefore, $B_{2}>A_{2}(\alpha)$ holds. 


\section{References}

Ausubel, L. M. (2000): "An Efficient Dynamic Auction for Heterogeneous Commodities," University of Maryland.

Demange, G. (1982): "Strategyproofness in the Assignment Market Game," Labratorie d'Econometrie de l'Ecole Polytechnique, Paris.

Gale, D., and L. Shapley (1962): "College Admissions and the Stability of Marriage," American Mathematical Monthly, 69, 9-15.

Gul, F., and E. Stacchetti (2000): "The English Auction with Differentiated Commodities," Journal of Economic Theory, 92(1), 95-124.

Herings, J. J. (1997): "A Globally and Universally Stable Price Adjustment Process," Journal of Mathematical Economics, 27, 163-193.

Hernando-Veciana, A. (2000): "Competition Among Auctioneers," Discussion paper, Unversidad de Alicante.

Kelso, A. S., and V. P. Crawford (1982): "Job Matching, Coalition Formation and Gross Substitutes," Econometrica, 50, 1483-1504.

Leonard, H. B. (1983): "Elicitation of Honest Preferences for the Assignment of Individuals to Positions," Journal of Political Economy, 91, 461-79.

McAfee, P. (1993): "Mechanism Design by Competing Sellers," Econometrica, 61(6), 1281-1312.

Morneau, J. (2001): "E-Marketplaces Start to Take Off," TechWeb News, http://www.techweb.com.

Myerson, R., and M. Satterthwaite (1983): "Efficient Mechanisms for Bilateral Trading," pp. 265-281.

Peters, M. (1997): "A Competitive Distribution of Auctions," Review of Economic Studies, 64, 97-124.

Peters, M., And S. Severinov (1997): "Competition Among Sellers who offer Auctions Instead of Prices," Journal of Economic Theory, 75(1), 141-179.

Roth, A., and A. Ockenfels (2000): "Last Minute Bidding and the Rules for Ending Second Price Auctions: Theory and Evidence from a Natural Experiment on the Internet," Discussion paper, Harvard University.

Roth, A. E., and M. A. Sotomayor (1990): Two-Sided Matching - A Study in Game Theoretic Modelling and Analysis, Econometric Society Monographs. Cambridge University Press, Cambridge MA.

Rubinstein, A., And A. Wolinsky (1990): "Decentralized Trading, Strategic Behaviour and the Walrasian Outcome," 57(189), 63-79. 
Rustichini, A., M. A. Satterthwaite, and S. R. Williams (1994): "Convergence to Efficiency in a Simple Market with Incomplete Information," Econometrica, 62(5), 1041-1063.

Satterthwaite, M. A., and S. Williams (1089): "The Rate of Convergence to Efficiency in the Buyer's bid Double Auction as the Market Becomes Large," 56, 477-498.

Smith, V. (1962): "An Experimental Study of Competitive Market Behavior," Journal of Political Economy.

Wilson, R. (1985): "Incentive Efficiency of Double Auctions," 53, 1101-1115.

Wilson, T., and T. Mullen (2000): "E-Business Exchanges Fight For Survival," InternetWeek, http://www.internetwk.com.

Zheng, M. (2001): "Bidding Behavior on Ebay," Discussion paper, University of Toronto, http://www.chass.utoronto.ca/ mzheng/papers/eBay.pdf. 